\title{
A Comparison of Populations Served by Kidney Paired Donation and List Paired Donation
}

\author{
Sommer E. Gentrya ${ }^{a}$, Dorry L. Segev ${ }^{b, *}$ \\ and Robert A. Montgomery \\ a Laboratory for Information and Decision Systems, \\ Massachusetts Institute of Technology, Cambridge, \\ Massachusetts, USA \\ ${ }^{\mathrm{b}}$ Department of Surgery, Johns Hopkins University School \\ of Medicine, Baltimore, Maryland, USA \\ * Corresponding author: Dorry L. Segev, dorry@jhmi.edu
}

\begin{abstract}
Options for utilizing live donor kidneys from those who are blood type incompatible or crossmatch positive with their intended recipients include kidney paired donation (KPD), list paired donation (LPD) and desensitization. KPD provides live donor kidneys for both recipients but requires a match to another incompatible pair, while LPD utilizes the deceased donor pool but is restricted by ethical and logistic concerns. We simulated patients and their potential donors to determine which recipients could receive a kidney through KPD and LPD. With smaller populations (100 pairs or fewer), more kidneys were matched through LPD, although the greatest benefit was derived from a combination of LPD and KPD. With increasing population sizes, more patients were matched through KPD, including almost all patients who would have been eligible for LPD. At population sizes predicted to be achieved by a national paired donation system, the role of LPD became minimal, with only $3.9 \%$ of pairs unmatched through KPD eligible for LPD. Considerable overlap was seen between the pairs unmatchable by KPD and those ineligible for LPD, namely less-demanded donors and hardto-match recipients. For this population, the best option may be desensitization.
\end{abstract}

Key words: Donor swaps, list exchange, paired kidney exchange

Received 03 March 2005, revised 26 March 2005 and accepted for publication 28 March 2005

\section{Introduction}

Currently, 60000 patients are waiting on the kidney transplant list and thousands more will be added this year (1). Very little increase has been seen in availability of deceased donor kidneys, while live donation has nearly tripled in the last decade. It is predicted that more than 2150 donor/recipient pairs per year are excluded from transplan- tation because of blood type incompatibility (ABOi) or positive crossmatch (+XM) (2).

Four options currently exist for incompatible donor/ recipient pairs. First, a number of specialized centers report success with incompatible kidney transplantation using desensitization strategies like plasmapheresis, intravenous immunoglobulin, pharmacologic B-cell depletion or splenectomy (3-13). Second, kidney paired donation (KPD) allows for exchange of donor organs between 2 incompatible donor/recipient pairs, such that the resulting pairs achieve compatibility (14-20). Third, list paired donation (LPD), also known as living-donor-cadaver-donor exchange or list exchange, allows the recipient of an incompatible pair to receive priority on the deceased donor list for providing the waitlist with the kidney from his intended live donor (21). The final option is to forgo live donation and wait on the deceased donor waiting list.

Most medically eligible incompatible pairs can theoretically be transplanted using desensitization protocols. Some patients will harbor very high levels of anti-HLA antibody against their donor, making desensitization impractical. The long-term graft and patient survival results of the various desensitization protocols are not yet known (22). In contrast, KPD can eliminate the incompatibilities between the matched pairs with results expected to recapitulate standard live donor transplants (15). However, not all patients can be matched through KPD $(23,24)$.

In LPD, a recipient trades off the disadvantage of accepting a deceased donor kidney (instead of the kidney from his live donor) with the advantage of avoiding incompatible transplantation (21). Since LPD selects exchanges from the deceased donor organ pool, it has proven useful for centers or regions where the breadth of deceased donors exceeds that of incompatible donors (21). However, major concerns have been raised about the equity of LPD. First, a deceased donor kidney has a considerably shorter halflife when compared to a live donor kidney (25). Furthermore, most of the recipients who present with an $A B O$ incompatible donor are blood type $\mathrm{O}$ and can only receive an $O$ kidney. For each LPD in which the deceased donor pool loses an O kidney and gains a non-O kidney, non-O waiting time on the list decreases while $O$ waiting time on the list increases (2). This disproportionately disadvantages blood type $\mathrm{O}$ patients on the waiting list who already endure longer average waiting times (2). This has led to calls 
for limiting donor/recipient pairs eligible for LPD, by over $50 \%$, in order to avoid harm to the waiting list (26).

We hypothesize that the most efficient and cost effective way to transplant an incompatible donor/recipient pair is through KPD. We have previously reviewed the cost benefit of KPD and the utility of an optimized algorithm for matching (23). In this manuscript, we simulate the blood types and sensitization of incompatible donor/recipient pairs, and characterize which pairs will be eligible and ineligible for LPD and which pairs will successfully match or remain unmatched after KPD. We show that LPD offers more opportunities than KPD when very small groups of patients are considered. This is likely the driving force behind LPD in small local or regional settings. As the incompatible population size increases, almost all patients who would have been eligible for LPD are matched via KPD, and the role for LPD nearly disappears at population sizes predicted to be achieved by a national paired donation system.

\section{Methods}

Since there are no direct data regarding incompatible donor/recipient pairs who would be considered for KPD or LPD, we simulated patient pools using United Network for Organ Sharing (UNOS) data and a decision tree model similar to ones previously described $(1,2,23,26)$.

\section{Simulated donors and recipients}

Simulated families begin with the intended recipient and his two parents. The potential donor pool includes the parents, siblings of the recipient, spouse, friends and children of the recipient (as many as 4 of which are eventually worked up). Parents are assigned a race based on UNOS kidney donor racial distributions (2). The recipient, his siblings and his children are assigned the same race as the parents. Race of unrelated potential donors is modeled as previously described (23). Blood types and HLA of the parents, spouse and unrelated donors are simulated based on race-specific $A B O$ (2) and HLA (27) distributions. The recipient, his siblings and his children are assigned $\mathrm{ABO}$ type and $\mathrm{HLA}$ by genetic inheritance.

\section{Decision tree model}

Up to 4 potential donors are selected for workup using a decision tree model similar to the one previously described $(2,26)$. The relationship to the recipient of the simulated potential donors is based on UNOS live kidney donor distribution (Table 1) (1). Medical ineligibility or unwillingness excludes $25 \%$ of spousal donors and $56.7 \%$ of other donors (2). Crossmatch tests are simulated based on recipient sensitization to HLA antigens. General sensitization of the recipient to HLA (as a result of blood transfusions,

Table 1: Donor-recipient relationships utilized in simulated decision tree model, as adapted from UNOS data (1)

\begin{tabular}{ll}
\hline Relationship of donor & $\%$ \\
\hline Parent & 19.7 \\
Child & 16.8 \\
Sibling & 42.4 \\
Spouse & 10.0 \\
Unrelated & 11.2 \\
\hline
\end{tabular}

childbirth or prior transplantation) is modeled by panel-reactive antibody (PRA), using a weighted combination of the most recent UNOS PRA distributions for waitlist additions and transplants (Table 2) (1). Furthermore, the increased sensitization of female recipients to their husbands and children is considered. Table 2 shows the general and wife/mother rates of positive crossmatch simulated for our decision tree model based on PRA assignments. After all exclusion criteria are considered, recipients with any willing, medically eligible, blood type compatible, crossmatch negative donors are assumed to undergo direct donation. Remaining recipients with a medically eligible, willing donor who is ABOi or +XM are considered for KPD or LPD.

\section{Estimated number of incompatible pairs per year}

In 2003 in the United States, 6468 live donor kidney transplants were performed (1). To determine the number of incompatible pairs that would arise nationally based on donor availability, we simulated recipients and donors using the applicable decision tree model until 6468 direct donor transplants were realized. Since this number varies with each execution of the decision tree model, a rounded mean was obtained over 30 runs and used to generate subsequent populations that were either entered into a KPD algorithm, LPD or a combination of KPD followed by LPD of eligible pairs unmatched by KPD.

\section{Kidney paired donation}

To determine the characteristics of patients that could be served by KPD, optimized KPD matching was performed using graph theory as previously described (23). In brief, a matching procedure was implemented on a personal computer based on the Edmonds algorithm $(28,29)$. Through this algorithm, every possible combination of matches from the donor/recipient pool was considered, compared and the combination that yielded the maximum number and quality of transplants was selected.

\section{List paired donation}

Pairs were considered ineligible for LPD based on the published exclusion criteria of the New England Organ Bank (21), an existing list exchange system, as well as ethical considerations outlined by Ross and Zenios (30). As such, excluded pairs were type O recipients with non-type-O donors (30) and highly sensitized recipients (PRA $>80 \%$ ) (21).

\section{Statistics}

For every experiment, we generated random databases of donor/recipient pairs, based on the simulated patient characteristics described above. Each experiment was executed 30 different times, each time using a newly simulated patient database. Statistical significance between numbers of pairs matched was calculated using Student's $t$-test.

\section{Results}

\section{Blood type distributions}

We simulated populations of incompatible donor/recipient pairs based on the assumption that each recipient had 2 potential donors (2-donor decision tree model). This simulation predicts a rounded mean of 3584 incompatible pairs per year with blood type distributions as shown (Table 3). Based on these characteristics, 30 separate donor/recipient populations were simulated. Each population in its entirety was reviewed for eligibility for LPD based on the published criteria $(21,30)$. Furthermore, each population in its entirety was entered into an optimized KPD algorithm (23). The blood type distributions of the 1330 


\section{Gentry et al.}

Table 2: Panel-reactive antibody (PRA) distributions, adapted from UNOS data (1), and simulated crossmatch likelihood based on PRA group

\begin{tabular}{lllllll}
\hline $\begin{array}{l}\text { PRA } \\
\text { group }\end{array}$ & $\begin{array}{l}\text { Awaiting } \\
\text { transplantation (\%) }\end{array}$ & $\begin{array}{l}\text { Deceased donor } \\
\text { recipients (\%) }\end{array}$ & $\begin{array}{l}\text { Live donor } \\
\text { recipients (\%) }\end{array}$ & $\begin{array}{l}\text { Overall weighted } \\
\text { distribution (\%)* }\end{array}$ & $\begin{array}{l}\text { Rate of positive } \\
\text { XM (\%) }\end{array}$ & $\begin{array}{l}\text { Rate of positive } \\
\text { XM for wife/mother (\%)** }\end{array}$ \\
\hline $0-9$ & 63.7 & 84.5 & 90.3 & 72.4 & 5 & 25 \\
$10-79$ & 21.2 & 11.6 & 8.5 & 17.1 & 45 & 65 \\
$80+$ & 15.1 & 3.9 & 1.2 & 10.5 & 90 & 95 \\
\hline
\end{tabular}

*Weighted distribution based on 23665 patients added to the waitlist in 2002, 8146 deceased donor recipients and 5931 live donor recipients. A weighted distribution was used to offset the fact that highly sensitized patients are more likely to wait on the waiting list. This simulation seeks to model all recipients who enter the system within a year, including those who undergo live donor transplantation, those who receive a deceased donor kidney and those who, for reasons of donor unavailability or HLA/ABO incompatibility, cannot be transplanted and are added to the waiting list.

**Multiparous female patients have an increased sensitization to their husbands as well as their children, in addition to any PRA they may have developed. For example, 2 random people have an $11.1 \%$ chance of a positive crossmatch between them, while a multiparous woman has a $33.3 \%$ chance of a positive crossmatch with her husband (2). For these crossmatches, we compound the positive crossmatch rate resulting from the woman's PRA with this added factor.

Table 3: Blood type distribution of 3584 incompatible donor/recipient pairs accrued over 1 year. Uses 2-donor decision tree model. dX denotes donor blood type and $r X$ denotes recipient blood type

\begin{tabular}{|c|c|c|c|c|c|c|c|c|c|c|c|}
\hline $\begin{array}{l}\text { Number of } \\
\text { pairs }\end{array}$ & $\mathrm{dO}$ & $d A$ & $\mathrm{~dB}$ & $d A B$ & Total & $\%$ & $\mathrm{dO}$ & $d A$ & $\mathrm{~dB}$ & $d A B$ & Total \\
\hline $\mathrm{rO}$ & 657 & 1066 & 354 & 51 & 2128 & $\mathrm{rO}$ & $18 \%$ & $30 \%$ & $10 \%$ & $1 \%$ & $59 \%$ \\
\hline rA & 257 & 429 & 174 & 107 & 967 & $r A$ & $7 \%$ & $12 \%$ & $5 \%$ & $3 \%$ & $27 \%$ \\
\hline$r B$ & 83 & 175 & 91 & 72 & 422 & $r B$ & $2 \%$ & $5 \%$ & $3 \%$ & $2 \%$ & $12 \%$ \\
\hline $\mathrm{rAB}$ & 12 & 27 & 17 & 11 & 67 & $r A B$ & $0 \%$ & $1 \%$ & $0 \%$ & $0 \%$ & $2 \%$ \\
\hline Total & 1010 & 1697 & 636 & 241 & 3584 & Total & $28 \%$ & $47 \%$ & $18 \%$ & $7 \%$ & \\
\hline
\end{tabular}

Table 4: Patients from Table 3 eligible for LPD

\begin{tabular}{|c|c|c|c|c|c|c|c|c|c|c|c|}
\hline $\begin{array}{l}\text { Number of } \\
\text { pairs }\end{array}$ & $\mathrm{dO}$ & $d A$ & $\mathrm{~dB}$ & $d A B$ & Total & $\%$ & $\mathrm{dO}$ & $d A$ & $\mathrm{~dB}$ & $d A B$ & Total \\
\hline rO & 363 & 0 & 0 & 0 & 363 & $\mathrm{rO}$ & $27 \%$ & $0 \%$ & $0 \%$ & $0 \%$ & $27 \%$ \\
\hline rA & 141 & 229 & 151 & 94 & 615 & $r A$ & $11 \%$ & $17 \%$ & $11 \%$ & $7 \%$ & $46 \%$ \\
\hline rB & 48 & 153 & 49 & 63 & 314 & $\mathrm{rB}$ & $4 \%$ & $12 \%$ & $4 \%$ & $5 \%$ & $24 \%$ \\
\hline $\mathrm{rAB}$ & 7 & 15 & 10 & 6 & 38 & $r A B$ & $1 \%$ & $1 \%$ & $1 \%$ & $0 \%$ & $3 \%$ \\
\hline Total & 559 & 398 & 209 & 163 & 1330 & Total & $42 \%$ & $30 \%$ & $16 \%$ & $12 \%$ & \\
\hline
\end{tabular}

Table 5: Patients from Table 3 who matched through optimized KPD

\begin{tabular}{|c|c|c|c|c|c|c|c|c|c|c|c|}
\hline $\begin{array}{l}\text { Number of } \\
\text { pairs }\end{array}$ & $\mathrm{dO}$ & $\mathrm{dA}$ & $\mathrm{dB}$ & $\mathrm{d} A \mathrm{~B}$ & Total & $\%$ & $\mathrm{dO}$ & $\mathrm{dA}$ & $\mathrm{dB}$ & $d A B$ & Total \\
\hline $\mathrm{rO}$ & 444 & 217 & 61 & 0 & 722 & $\mathrm{rO}$ & $26 \%$ & $13 \%$ & $3 \%$ & $0 \%$ & $42 \%$ \\
\hline$r A$ & 232 & 274 & 151 & 17 & 674 & rA & $13 \%$ & $16 \%$ & $9 \%$ & $1 \%$ & $39 \%$ \\
\hline$r B$ & 70 & 153 & 52 & 10 & 285 & $\mathrm{rB}$ & $4 \%$ & $9 \%$ & $3 \%$ & $1 \%$ & $16 \%$ \\
\hline$r A B$ & 11 & 20 & 12 & 5 & 49 & $\mathrm{rAB}$ & $1 \%$ & $1 \%$ & $1 \%$ & $0 \%$ & $3 \%$ \\
\hline Total & 756 & 665 & 276 & 33 & 1730 & Total & $44 \%$ & $38 \%$ & $16 \%$ & $2 \%$ & \\
\hline
\end{tabular}

pairs (37\% of original population) eligible for list exchange (Table 4), the 1730 pairs (48\% of original population) who matched through KPD (Table 5) and the 1854 pairs (52\% of the original population) who remained unmatched after KPD (Table 6) are compared. Patients unmatched after KPD were then reviewed for eligibility for LPD (Table 7).

Blood-type-specific inequities are seen in the UNOS data and further corroborated by our findings. The scarcity of donor availability to type $O$ recipients is evidenced by the fact that $53 \%$ of the waiting list is type O, while only $46 \%$ of transplant recipients are type $\mathrm{O}$. Conversely, it is clear that type A recipients have a broader supply of organs, because $28 \%$ of the waitlist is type A while $37 \%$ of recipients are type $A(1)$. Similarly, populations of incompatible kidney pairs who do not match after KPD (i.e. the 'KPD waiting list') are heavily weighted to type $O$ recipients (76\%) and underrepresented by their type A counterparts (16\%) when 
Table 6: Patients from Table 3 who remain unmatched after KPD

\begin{tabular}{|c|c|c|c|c|c|c|c|c|c|c|c|}
\hline $\begin{array}{l}\text { Number of } \\
\text { pairs }\end{array}$ & $\mathrm{dO}$ & $\mathrm{dA}$ & $\mathrm{dB}$ & $d A B$ & Total & $\%$ & $\mathrm{dO}$ & $\mathrm{dA}$ & $\mathrm{dB}$ & $d A B$ & Total \\
\hline $\mathrm{rO}$ & 214 & 849 & 293 & 50 & 1406 & $\mathrm{rO}$ & $12 \%$ & $46 \%$ & $16 \%$ & $3 \%$ & $76 \%$ \\
\hline rA & 26 & 155 & 23 & 90 & 293 & rA & $1 \%$ & $8 \%$ & $1 \%$ & $5 \%$ & $16 \%$ \\
\hline$r B$ & 14 & 22 & 39 & 62 & 137 & $r B$ & $1 \%$ & $1 \%$ & $2 \%$ & $3 \%$ & $7 \%$ \\
\hline$r A B$ & 1 & 7 & 5 & 6 & 18 & $\mathrm{rAB}$ & $0 \%$ & $0 \%$ & $0 \%$ & $0 \%$ & $1 \%$ \\
\hline Total & 254 & 1032 & 360 & 208 & 1854 & Total & $14 \%$ & $56 \%$ & $19 \%$ & $11 \%$ & \\
\hline
\end{tabular}

Table 7: Patients from Table 6 eligible for LPD

\begin{tabular}{|c|c|c|c|c|c|c|c|c|c|c|c|}
\hline $\begin{array}{l}\text { Number of } \\
\text { pairs }\end{array}$ & $\mathrm{dO}$ & $\mathrm{dA}$ & $\mathrm{dB}$ & $\mathrm{dAB}$ & Total & $\%$ & $\mathrm{dO}$ & $\mathrm{dA}$ & $\mathrm{dB}$ & $\mathrm{d} A \mathrm{~B}$ & Total \\
\hline $\mathrm{rO}$ & 0 & 0 & 0 & 0 & 0 & $\mathrm{rO}$ & $0 \%$ & $0 \%$ & $0 \%$ & $0 \%$ & $0 \%$ \\
\hline rA & 0 & 0 & 4 & 77 & 82 & $r A$ & $0 \%$ & $0 \%$ & $3 \%$ & $55 \%$ & $58 \%$ \\
\hline rB & 0 & 5 & 0 & 53 & 59 & $\mathrm{rB}$ & $0 \%$ & $4 \%$ & $0 \%$ & $38 \%$ & $42 \%$ \\
\hline$r A B$ & 0 & 0 & 0 & 0 & 0 & $\mathrm{rAB}$ & $0 \%$ & $0 \%$ & $0 \%$ & $0 \%$ & $0 \%$ \\
\hline Total & 0 & 5 & 5 & 131 & 141 & Total & $0 \%$ & $4 \%$ & $3 \%$ & $93 \%$ & \\
\hline
\end{tabular}

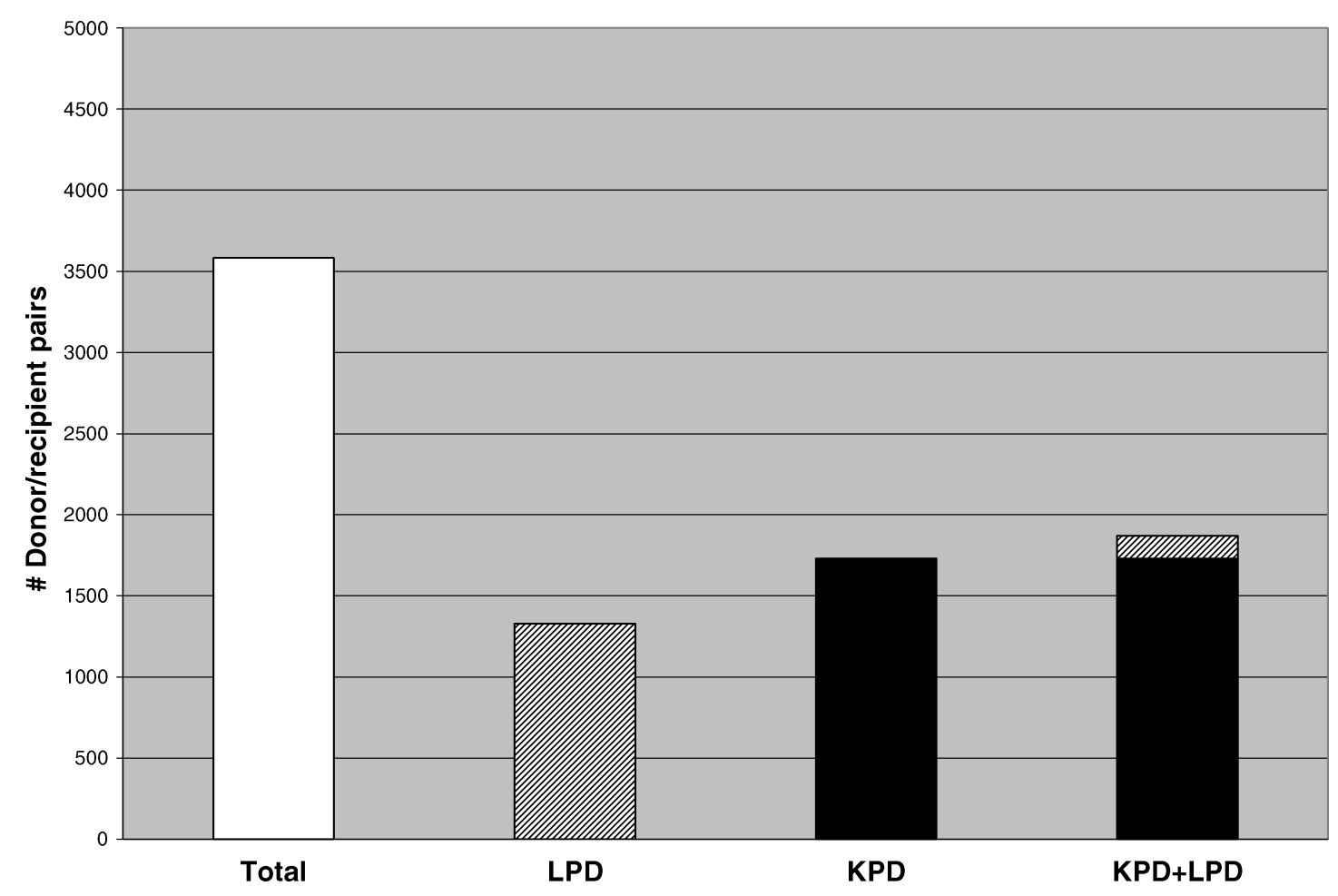

Figure 1: Potential transplants resulting from list paired donation (LPD), kidney paired donation (KPD) and KPD followed by LPD of unmatched pairs. Uses 2-donor decision tree model.

compared with patients who are matched (42\% type $\mathrm{O}$ and $39 \%$ type A). Just as the majority of the waitlist (i.e. patients yet unmatched to deceased donors) is comprised of type $\mathrm{O}$ recipients, most of the patients unmatched through KPD are also type $O$, with type A donors. Since the type $O$ donor shortage makes these pairs ethically ineligible for LPD (26), the role for LPD in the setting of KPD is limited.

\section{Relative contributions of KPD and LPD}

For the 2-donor decision tree model simulated above, we compared the number of potential transplants resulting from LPD alone, KPD alone or KPD followed by LPD for patients unmatched by KPD (Figure 1). The relative contributions of KPD and LPD to a combined approach are also delineated. For this population, KPD leads to successful transplantation for more patients than LPD (1730 vs. 1330, 


\section{Gentry et al.}

Table 8: Blood type distribution (\%) for varying population sizes based on characteristics of a 2-donor decision tree model. $d X$ denotes donor blood type and $r X$ denotes recipient blood type

\begin{tabular}{|c|c|c|c|c|c|c|c|c|c|}
\hline & \multicolumn{3}{|c|}{25 pairs } & \multicolumn{3}{|c|}{250 pairs } & \multicolumn{3}{|c|}{2500 pairs } \\
\hline & LPD & KPD & Post-LPD & LPD & KPD & Post-LPD & LPD & KPD & Post-LPD \\
\hline Pairs/year & 9 & 7 & 3 & 93 & 98 & 14 & 928 & 1170 & 101 \\
\hline $\mathrm{dO} \mathrm{rO}$ & $29.1 \%$ & $33.0 \%$ & $10.7 \%$ & $28.7 \%$ & $28.1 \%$ & $2.4 \%$ & $27.4 \%$ & $25.6 \%$ & $0.1 \%$ \\
\hline $\mathrm{dA}$ rO & $0.0 \%$ & $11.8 \%$ & $0.0 \%$ & $0.0 \%$ & $11.1 \%$ & $0.0 \%$ & $0.0 \%$ & $12.2 \%$ & $0.0 \%$ \\
\hline $\mathrm{dB} \mathrm{rO}$ & $0.0 \%$ & $4.7 \%$ & $0.0 \%$ & $0.0 \%$ & $3.4 \%$ & $0.0 \%$ & $0.0 \%$ & $3.4 \%$ & $0.0 \%$ \\
\hline $\mathrm{dAB} r \mathrm{r}$ & $0.0 \%$ & $0.0 \%$ & $0.0 \%$ & $0.0 \%$ & $0.0 \%$ & $0.0 \%$ & $0.0 \%$ & $0.0 \%$ & $0.0 \%$ \\
\hline $\mathrm{dO}$ rA & $11.2 \%$ & $15.1 \%$ & $0.0 \%$ & $10.5 \%$ & $12.0 \%$ & $0.0 \%$ & $10.4 \%$ & $13.2 \%$ & $0.0 \%$ \\
\hline$d A r A$ & $11.5 \%$ & $10.4 \%$ & $10.7 \%$ & $17.8 \%$ & $17.3 \%$ & $3.4 \%$ & $17.7 \%$ & $16.3 \%$ & $0.1 \%$ \\
\hline $\mathrm{dB} r \mathrm{~A}$ & $15.8 \%$ & $9.0 \%$ & $24.3 \%$ & $10.8 \%$ & $9.2 \%$ & $7.5 \%$ & $11.0 \%$ & $8.9 \%$ & $1.0 \%$ \\
\hline$d A B$ rA & $6.8 \%$ & $0.5 \%$ & $17.5 \%$ & $6.8 \%$ & $0.8 \%$ & $39.7 \%$ & $7.2 \%$ & $0.9 \%$ & $56.5 \%$ \\
\hline $\mathrm{dO}$ rB & $3.6 \%$ & $4.7 \%$ & $0.0 \%$ & $3.3 \%$ & $3.5 \%$ & $0.0 \%$ & $3.5 \%$ & $3.9 \%$ & $0.0 \%$ \\
\hline$d A r B$ & $11.2 \%$ & $9.0 \%$ & $11.7 \%$ & $11.6 \%$ & $9.4 \%$ & $11.6 \%$ & $11.6 \%$ & $9.0 \%$ & $4.2 \%$ \\
\hline $\mathrm{dB} r \mathrm{~B}$ & $4.3 \%$ & $0.0 \%$ & $11.7 \%$ & $2.9 \%$ & $2.5 \%$ & $2.4 \%$ & $3.8 \%$ & $3.1 \%$ & $0.4 \%$ \\
\hline$d A B$ rB & $4.7 \%$ & $0.5 \%$ & $11.7 \%$ & $4.8 \%$ & $0.3 \%$ & $30.3 \%$ & $4.8 \%$ & $0.6 \%$ & $37.4 \%$ \\
\hline $\mathrm{dO} r \mathrm{rB}$ & $0.7 \%$ & $0.9 \%$ & $0.0 \%$ & $0.7 \%$ & $0.7 \%$ & $0.0 \%$ & $0.5 \%$ & $0.7 \%$ & $0.0 \%$ \\
\hline$d A$ rAB & $0.4 \%$ & $0.0 \%$ & $1.0 \%$ & $1.1 \%$ & $1.1 \%$ & $0.0 \%$ & $1.0 \%$ & $1.0 \%$ & $0.0 \%$ \\
\hline $\mathrm{dB} r \mathrm{AB}$ & $0.4 \%$ & $0.5 \%$ & $0.0 \%$ & $0.5 \%$ & $0.4 \%$ & $0.0 \%$ & $0.7 \%$ & $0.7 \%$ & $0.0 \%$ \\
\hline $\mathrm{dAB} r A B$ & $0.4 \%$ & $0.0 \%$ & $1.0 \%$ & $0.6 \%$ & $0.2 \%$ & $2.7 \%$ & $0.4 \%$ & $0.3 \%$ & $0.4 \%$ \\
\hline
\end{tabular}

$\mathrm{LPD}=$ entire population evaluated for eligibility for list paired donation; KPD = entire population entered into KPD, matched patients reported; Post-LPD = After KPD is performed, unmatched patients eligible for LPD are reported.

$p<0.0001)$. A small further gain is seen when patients unmatched by KPD are considered for LPD as well (141 eligible patients out of 3584 incompatible pairs).

\section{Effect of incompatible population size}

To evaluate the effect of incompatible population size on the role of LPD and KPD and the characteristics of the populations involved, we simulated populations of varying sizes using the blood type distributions of the 2-donor decision tree model. We report, for selected sample sizes, the blood type distributions of patients eligible for LPD, those who matched through KPD and those who remained unmatched after KPD but were eligible for LPD (Table 8). Furthermore, we compare the number of potential transplants resulting from LPD, KPD and KPD followed by LPD (Figure 2).

With very small populations of donor/recipient pairs (100 or fewer), more patients will be served by LPD than by KPD ( $p<0.05$ for 50 or fewer pairs, trend noted but not statistically significant for 100 pairs), because LPD can exchange with the entire deceased donor pool while KPD is limited to the incompatible pool. However, it is clear that regardless of donor makeup, more pairs will be served by a combined approach (KPD followed by LPD) than by either modality alone.

\section{Effect of potential donor pool}

For the decision tree model used in these simulations, the number of potential donors (before medical workup, psychological evaluation, blood typing and crossmatch are performed) who are available to each recipient is chosen. To evaluate the effect of donor availability on the role of
LPD and KPD and the characteristics of the populations involved, we simulated populations based on 1-, 2-, 3- and 4-donor decision tree models. We report, for each number of available donors, the blood type distributions of patients eligible for LPD, those who matched through KPD, and those who remained unmatched after KPD but were eligible for LPD (Table 9). Furthermore, we compare the number of potential transplants resulting from LPD, KPD and KPD followed by LPD (Figure 3). The available incompatible donor/recipient population decreases in size as number of potential donors increases because of the resulting increase in compatible donor availability. However, no matter how many potential donors are available (between 1 and 4), more patients are served by KPD than by LPD ( $p<$ 0.0001 ), with some added benefit to a combined approach (KPD followed by LPD).

\section{Discussion}

A kidney transplant recipient with an incompatible live donor has four choices: (i) undergo desensitization and receive the kidney directly from the live donor, (ii) agree to accept a deceased donor organ through a LPD, (iii) enter a KPD in an attempt to find a live donor kidney without the need for undergoing desensitization therapy or (iv) register for the deceased donor waiting list. As the availability of the first three modalities increases throughout the country, various questions and controversies have arisen regarding the most favorable approach in terms of access, equity, results and cost. In this report, we use a decision tree model simulation and optimized KPD algorithm to answer important questions relating to the relative merits and shortcomings of these three approaches. 


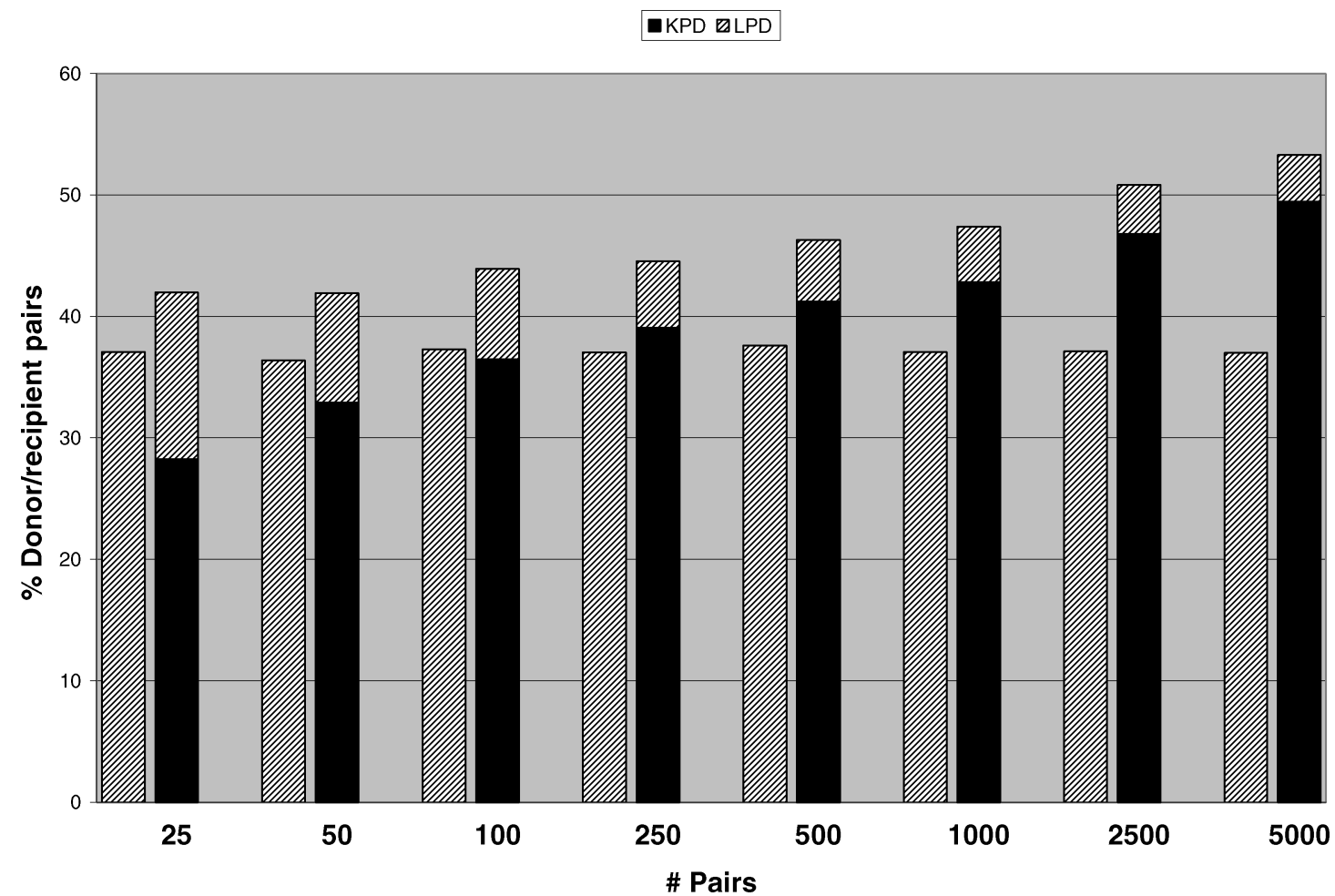

Figure 2: Populations served by list paired donation (LPD), kidney paired donation (KPD) and LPD following KPD for varying population sizes.

Table 9: Blood type distribution (\%) and estimated annual totals of incompatible donor/recipient pairs for varying numbers of potential donors. $\mathrm{dX}$ denotes donor blood type and $\mathrm{rX}$ denotes recipient blood type

\begin{tabular}{|c|c|c|c|c|c|c|c|c|c|c|c|c|}
\hline & \multicolumn{3}{|c|}{1 donor (4443 pairs) } & \multicolumn{3}{|c|}{2 donors (3584 pairs) } & \multicolumn{3}{|c|}{3 donors (2891 pairs) } & \multicolumn{3}{|c|}{4 donors (2406 pairs) } \\
\hline & LPD & KPD & Post-LPD & LPD & KPD & Post-LPD & LPD & KPD & Post-LPD & LPD & KPD & Post-LPD \\
\hline Pairs/year & 1731 & 2255 & 181 & 1334 & 1737 & 142 & 1000 & 1304 & 110 & 781 & 1030 & 87 \\
\hline $\mathrm{dO} \mathrm{rO}$ & $26.6 \%$ & $25.1 \%$ & $0.0 \%$ & $27.3 \%$ & $25.7 \%$ & $0.0 \%$ & $28.9 \%$ & $26.7 \%$ & $0.1 \%$ & $29.3 \%$ & $26.7 \%$ & $0.1 \%$ \\
\hline $\mathrm{dA}$ rO & $0.0 \%$ & $12.5 \%$ & $0.0 \%$ & $0.0 \%$ & $12.4 \%$ & $0.0 \%$ & $0.0 \%$ & $12.6 \%$ & $0.0 \%$ & $0.0 \%$ & $13.0 \%$ & $0.0 \%$ \\
\hline $\mathrm{dB}$ rO & $0.0 \%$ & $3.4 \%$ & $0.0 \%$ & $0.0 \%$ & $3.4 \%$ & $0.0 \%$ & $0.0 \%$ & $3.4 \%$ & $0.0 \%$ & $0.0 \%$ & $3.6 \%$ & $0.0 \%$ \\
\hline $\mathrm{dAB}$ rO & $0.0 \%$ & $0.0 \%$ & $0.0 \%$ & $0.0 \%$ & $0.0 \%$ & $0.0 \%$ & $0.0 \%$ & $0.0 \%$ & $0.0 \%$ & $0.0 \%$ & $0.0 \%$ & $0.0 \%$ \\
\hline dO rA & $10.9 \%$ & $13.1 \%$ & $0.0 \%$ & $10.3 \%$ & $13.3 \%$ & $0.0 \%$ & $10.3 \%$ & $13.8 \%$ & $0.0 \%$ & $10.7 \%$ & $14.3 \%$ & $0.0 \%$ \\
\hline $\mathrm{dA} r \mathrm{~A}$ & $17.7 \%$ & $16.5 \%$ & $0.0 \%$ & $17.5 \%$ & $16.1 \%$ & $0.0 \%$ & $17.2 \%$ & $15.5 \%$ & $0.1 \%$ & $16.5 \%$ & $15.0 \%$ & $0.2 \%$ \\
\hline$d B$ rA & $11.9 \%$ & $9.1 \%$ & $4.8 \%$ & $11.4 \%$ & $8.9 \%$ & $2.8 \%$ & $10.8 \%$ & $8.4 \%$ & $2.1 \%$ & $10.1 \%$ & $8.0 \%$ & $0.3 \%$ \\
\hline$d A B$ rA & $7.1 \%$ & $0.9 \%$ & $56.7 \%$ & $7.1 \%$ & $0.9 \%$ & $56.5 \%$ & $7.0 \%$ & $1.0 \%$ & $52.3 \%$ & $6.5 \%$ & $0.8 \%$ & $48.9 \%$ \\
\hline $\mathrm{dO}$ rB & $3.4 \%$ & $3.9 \%$ & $0.0 \%$ & $3.5 \%$ & $4.0 \%$ & $0.0 \%$ & $3.4 \%$ & $4.0 \%$ & $0.0 \%$ & $3.7 \%$ & $4.2 \%$ & $0.0 \%$ \\
\hline$d A r B$ & $11.4 \%$ & $9.0 \%$ & $1.6 \%$ & $11.5 \%$ & $8.9 \%$ & $2.0 \%$ & $11.4 \%$ & $8.6 \%$ & $5.0 \%$ & $11.8 \%$ & $8.3 \%$ & $9.8 \%$ \\
\hline$d B r B$ & $3.6 \%$ & $3.0 \%$ & $0.2 \%$ & $3.7 \%$ & $3.0 \%$ & $0.2 \%$ & $3.4 \%$ & $2.8 \%$ & $0.2 \%$ & $3.7 \%$ & $2.9 \%$ & $0.3 \%$ \\
\hline$d A B$ rB & $4.5 \%$ & $0.6 \%$ & $36.4 \%$ & $4.9 \%$ & $0.7 \%$ & $38.2 \%$ & $5.1 \%$ & $0.5 \%$ & $39.7 \%$ & $5.1 \%$ & $0.5 \%$ & $40.0 \%$ \\
\hline dO rAB & $0.5 \%$ & $0.6 \%$ & $0.0 \%$ & $0.5 \%$ & $0.6 \%$ & $0.0 \%$ & $0.4 \%$ & $0.6 \%$ & $0.0 \%$ & $0.5 \%$ & $0.8 \%$ & $0.0 \%$ \\
\hline$d A$ rAB & $1.1 \%$ & $1.2 \%$ & $0.0 \%$ & $1.0 \%$ & $1.1 \%$ & $0.0 \%$ & $1.1 \%$ & $1.2 \%$ & $0.0 \%$ & $1.0 \%$ & $1.0 \%$ & $0.0 \%$ \\
\hline $\mathrm{dB} r \mathrm{rB}$ & $0.7 \%$ & $0.7 \%$ & $0.0 \%$ & $0.7 \%$ & $0.8 \%$ & $0.0 \%$ & $0.7 \%$ & $0.6 \%$ & $0.0 \%$ & $0.6 \%$ & $0.7 \%$ & $0.0 \%$ \\
\hline $\mathrm{d} A \mathrm{~B}$ rAB & $0.4 \%$ & $0.3 \%$ & $0.2 \%$ & $0.5 \%$ & $0.4 \%$ & $0.2 \%$ & $0.5 \%$ & $0.4 \%$ & $0.4 \%$ & $0.4 \%$ & $0.3 \%$ & $0.3 \%$ \\
\hline
\end{tabular}

$\mathrm{LPD}=$ entire population evaluated for eligibility for list paired exchange; $\mathrm{KPD}=$ entire population entered into KPD, matched patients reported; Post-LPD = after KPD is performed, unmatched patients eligible for LPD are reported.

The results from a limited number of institutions employing desensitization protocols are encouraging but long-term results are not yet available and therapy is labor intensive, immunosuppressive and costly (an average course of plasmapheresis/low-dose IVIg adds $\$ 28000$ to the transplant event) (31). Still this approach will undoubtedly be used for difficult-to-match recipients (e.g. blood type O, low donor-specific antibody titer or broadly sensitized 


\section{Gentry et al.}

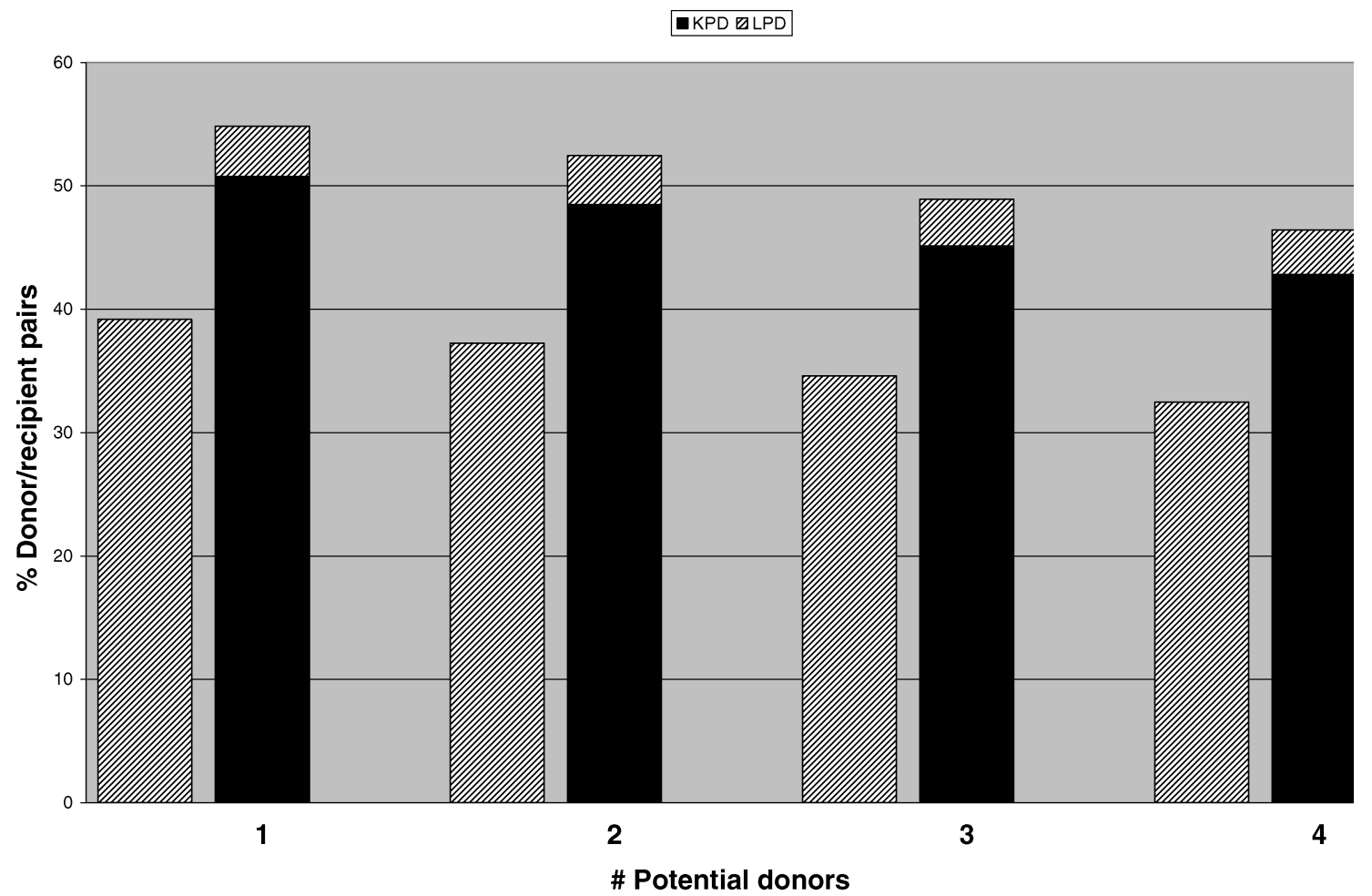

Figure 3: Populations served by list paired donation (LPD), kidney paired donation (KPD) and LPD following KPD for varying numbers of potential donors.

recipients). Patients who remain unmatched after KPD could enter a second pool in which a more immunologically compatible donor is sought but the requirement for $\mathrm{ABO}$ compatibility or crossmatch negativity is relaxed. The goal in this incompatible paired donation would be to reduce the intensity and cost of desensitization. Alternatively, unmatched patients could wait for other pairs to enter the pool.

LPD offers patients with incompatible live donors the option of moving to the top of the deceased donor waiting list in return for providing a live donor kidney to the deceased donor pool. We have shown that for small populations, more patients are served by LPD than KPD. This is consistent with a report from New England, where both KPD and LPD are in practice, and that 17 patients were transplanted via LPD when compared with 8 through KPD. However, ethical concerns may prevent $\mathrm{O}$ recipients with non-O donors from undergoing LPD (30). No such restrictions are necessary for live donor KPD because the deceased donor list is not adversely affected.

We have shown that the greatest number of matches can be achieved through a national paired donation program utilizing an optimized algorithm for patients who have eligible incompatible live donors (23). Using simulated populations of incompatible pairs, we have shown that on a national level, as a primary modality, LPD will yield fewer matches and is much less desirable than a national KPD. Combining LPD with KPD will only yield a very small number of additional matches over what can be achieved by KPD alone. LPD after KPD may be ethically flawed, because LPD of nearly all of the patients unmatched after KPD would likely worsen the inequity in waiting time on the list. Since KPD provides both matched recipients with a more durable live donor kidney and is free of the ethical concerns surrounding LPD, we feel that LPD at best has a limited role on a national level. For patients unmatched by KPD and ineligible for LPD, desensitization likely offers the best chance for success.

\section{References}

1. UNOS. Organ Procurement and Transplantation Network data as of Sept 17, obtained from and available at the United Network for Organ Sharing web site. 2004.

2. Zenios SA, Woodle ES, Ross LF. Primum non nocere: avoiding harm to vulnerable wait list candidates in an indirect kidney exchange. Transplantation 2001; 72: 648-654.

3. Montgomery RA, Zachary AA, Racusen LC et al. Plasmapheresis and intravenous immune globulin provides effective rescue therapy for refractory humoral rejection and allows kidneys to be successfully transplanted into cross-match-positive recipients. Transplantation 2000; 70: 887-895.

4. Alkhunaizi AM, de Mattos AM, Barry JM, Bennett WM, Norman DJ. Renal transplantation across the $\mathrm{ABO}$ barrier using $\mathrm{A} 2$ kidneys. Transplantation 1999; 67: 1319-1324. 
5. Gloor JM, Lager DJ, Moore SB et al. ABO-incompatible kidney transplantation using both $\mathrm{A} 2$ and non-A2 living donors. Transplantation 2003; 75: 971-977.

6. Montgomery RA, Cooper M, Kraus E et al. Renal transplantation at the Johns Hopkins Comprehensive Transplant Center. Clin Transpl 2003:199-213.

7. Sawada T, Fuchinoue S, Teraoka S. Successful A1-to-O ABOincompatible kidney transplantation after a preconditioning regimen consisting of anti-CD20 monoclonal antibody infusions, splenectomy, and double-filtration plasmapheresis. Transplantation 2002; 74: 1207-1210.

8. Sonnenday CJ, Ratner LE, Zachary AA et al. Preemptive therapy with plasmapheresis/intravenous immunoglobulin allows successful live donor renal transplantation in patients with a positive cross-match. Transplant Proc 2002; 34: 1614-1616.

9. Sonnenday CJ, Warren DS, Cooper M et al. Plasmapheresis, CMV hyperimmune globulin, and anti-CD20 allow ABO-incompatible renal transplantation without splenectomy. Am J Transplant 2004; 4: 1315-1322

10. Sorensen JB, Grant WJ, Belnap LP, Stinson J, Fuller TC. Transplantation of $\mathrm{ABO}$ group $\mathrm{A} 2$ kidneys from living donors into group $\mathrm{O}$ and B recipients. Am J Transplant 2001; 1: 296-299.

11. Takahashi K, Saito K, Takahara $S$ et al. Excellent long-term outcome of $\mathrm{ABO}$-incompatible living donor kidney transplantation in Japan. Am J Transplant 2004; 4: 1089-1096.

12. Tanabe K, Takahashi K, Sonda K et al. Long-term results of ABOincompatible living kidney transplantation: a single-center experience. Transplantation 1998; 65: 224-228.

13. Tyden G, Kumlien G, Fehrman I. Successful ABO-incompatible kidney transplantations without splenectomy using antigenspecific immunoadsorption and rituximab. Transplantation 2003; 76: 730-731.

14. Rapaport FT. The case for a living emotionally related international kidney donor exchange registry. Transplant Proc 1986; 18(Suppl. 2):5-9.

15. Montgomery RA, Zachary AA, Ratner LE et al. Kidney paired donation permits successful transplantation with incompatible live kidney donors. Submitted.

16. Ross LF, Rubin DT, Siegler M, Josephson MA, Thistlethwaite JR,
Jr., Woodle ES. Ethics of a paired-kidney-exchange program. N Engl J Med 1997; 336: 1752-1755.

17. Ross LF, Woodle ES. Ethical issues in increasing living kidney donations by expanding kidney paired exchange programs. Transplantation 2000; 69: 1539-1543.

18. Sells RA. Paired-kidney-exchange programs. N Engl J Med 1997; 337: 1392-1393.

19. de Klerk M, Keizer K, Weimar W. Donor exchange for renal transplantation. N Engl J Med 2004; 351: 935-937; author reply 935937.

20. Kranenburg LW, Visak T, Weimar W et al. Starting a crossover kidney transplantation program in the Netherlands: ethical and psychological considerations. Transplantation 2004; 78: 194-197.

21. Delmonico FL, Morrissey PE, Lipkowitz GS et al. Donor kidney exchanges. Am J Transplant 2004; 4: 1628-1634.

22. Stegall MD, Dean PG, Gloor JM. ABO-incompatible kidney transplantation. Transplantation 2004; 78: 635-640.

23. Segev DL, Gentry SE, Warren DS, Reeb B, Montgomery RA. Kidney paired donation and optimizing the use of live donor organs. JAMA 2005; 293: 1883-1890.

24. Roth $A E$, Sönmez T, Ünver MU. Kidney exchange. Q J Econ 2004:457-488

25. Terasaki PI, Cecka JM, Gjertson DW, Takemoto S. High survival rates of kidney transplants from spousal and living unrelated donors. N Engl J Med 1995; 333: 333-336.

26. Ross LF, Zenios S. Restricting living-donor-cadaver-donor exchanges to ensure that standard blood type o wait-list candidates benefit. Transplantation 2004; 78: 641-646.

27. Leffell MS, Steinberg AG, Bias WB, Machan CH, Zachary AA. The distribution of HLA antigens and phenotypes among donors and patients in the UNOS registry. Transplantation 1994; 58: 11191130.

28. Edmonds J. Paths, trees, and flowers. Can J Math 1965; 17: 449467.

29. Lovász L, Plummer MD. Matching Theory. New York: Elsevier, 1986: 369-375.

30. Ross LF, Zenios S. Practical and ethical challenges to paired exchange programs. Am J Transplant 2004; 4: 1553-1544.

31. USRDS. United States Renal Data Systems, Annual Report. 2003. 